\title{
Enrichment of Molybdenum and Fluorite by Flotation of Fluorite Ore Containing Molybdenum
}

\author{
Abuzer Akgün, İbrahim Teğin, Recep Ziyadanoğulları* \\ Department of Chemistry, Faculty of Science and Art, Dicle University, \\ 21280 Diyarbakir, TURKEY \\ *For correspondence: Fax: +90-412-2488039 Tel : +90-412-2488550/3159 E-mail: recepz@dicle.edu.tr
}

\begin{abstract}
The molybdenum and fluorite were obtained in different phases with the flotation of fluorite ore containing molybdenum obtained from Karaamă̆aradere-Keban-Elazlğ district in Turkey. It has been found that the original ore contains $1.08 \% \mathrm{Mo}, 0.05 \% \mathrm{Cu}, 1.20 \% \mathrm{~Pb}, 1.12 \% \mathrm{Zn}$ and $12.0 \% \mathrm{~F}$. It was determined that molybdenum and fluorite could not be enriched in different phases with satisfactory yields by flotation of original ore at the defined size. Thus, the ore was sulfurized before the flotation. Concentrates containing molybdenum and the other concentrates containing fluorite were collected with fairly high yields in different phases by the flotation of the sulfurized ore. In the optimum flotation conditions, the $\mathrm{Mo}, \mathrm{Pb}, \mathrm{Cu}$ and $\mathrm{Zn}$ were obtained over 95 $\%$ yields and approximately $6 \%$ fluorite passed into concentrate phase. Mo and $\mathrm{Cu}$ containing phase were enriched with high yield by selective flotation of concentrate obtained from the concentrate. On the other hand, the most of $\mathrm{Pb}$ and $\mathrm{Zn}$ remained in tailing. In order to gain molybdenum, the appropriate concentrate was obtained by this process with hydrometallurgical method. It was also determined that the trace amounts of $\mathrm{Ta}, \mathrm{Nb}$ and $\mathrm{V}$ in ore were collected in the concentrate phase.

In the first step of flotation, the fluorite left in the tailing contained $29.6 \%$ of CaF $\mathrm{F}_{2}$ After flotation of this tailing, concentrate grade of $\mathrm{CaF}_{2}$ raised to $96 \% .0 .5 \%$ of sulfur was found in this concentrate. It was concluded that this concentrate contained fluorite with suitable particle size (-100 mesh) and at sufficient concentration for metallurgical processes.
\end{abstract}

Key Words: Molybdenum, fluorite, sulfurization, flotation 


\section{INTRODUCTION}

Fluorite $\left(\mathrm{CaF}_{2}\right)$ is an important fluorine mineral, which is mostly used for the production of hydrofluoric acid and as a flux in steel making. Other uses are the manufacture of glass, fiberglass, pottery and enamel, etc.

Recently, there have been numerous reports on the modification of the chemical scheme in fluorite flotation, in order to increase the separation efficiency and lower the operation costs.

It was [1] reported that methanoic acid could selectively adsorb onto fluorite surfaces in preference to calcite surfaces at ambient temperature. This means that fluorite flotation to remove calcite might be achieved at a much lower temperature if methanoic acid is used as collector, resulting in a large reduction in energy consumption. It was $[2,3]$ found that sodium $\mathrm{N}$-dodecanoyl sarcosine (anionic collector) in combination with dodecylammonium chloride (cationic collector) as co-collectors could greatly enhance the floatability of fluorite, compared with the use of the anionic collector alone. Also, fluorite flotation could be improved by using effective depressants when fatty acid is used as collector. For example, acidized sodium silicate was reported to depress calcite strongly in preference of fluorite at ambient temperature, leading the fluorite flotation to be achieved at a lower temperature [4].

Molybdenum and fluorite are important materials for modern industry. Therefore, production of fluorite and molybdenum from fluorite ore containing molybdenum are significant. The most important minerals of molybdenum are molybdenite $\left(\mathrm{MoS}_{2}\right)$, wulpherite $\left(\mathrm{PbMoO}_{4}\right)$, and molybdite $\left(\mathrm{MoO}_{3}\right)$. However, for molybdenum production from ores containing molybdenum in the first step, enrichment is required. Therefore, the flotation method was applied. Molybdenum usually occurs as molybdenite in nature and enrichment of molybdenum is much easier in comparison to other ores with high yield. Molybdenum has different constituent in minerals. Therefore, it was determined that flotation yields are low. Moreover, recovery of concentrate of molybdenum and fluorite separately reduce flotation yield [5].

Flotation tests on fluorite ores with various carbonate content $\left(\mathrm{CaCO}_{3}\right.$ contents from $4.43 \%$ to $74.40 \%$ ) were carried out using acidized sodium silicate as modifier and oleic acid as collector. A high purity fluorite concentrate $\left(99 \% \mathrm{CaF}_{2}\right)$ was obtained. It was demonstrated that acidized sodium silicate has an activating effect on fluorite and a selective depressing effect on calcite in rougher flotation, at the same time effectively removing the floated calcite from the rougher concentrate [6].

In this study, we aimed to collect molybdenum and fluorite in separate phases and to increase flotation yield. Therefore, before the flotation, the sulfurization process was made in order 
to change the ore structure and surface properties [7, 8]. Sulfurized sample were subjected to flotation and results were evaluated [9].

\section{MATERIALS AND METHOD}

\subsection{Materials}

The fluorite ore containing molybdenum used in this study was obtained from Karamağaradere district of Keban-Elazı $\breve{g}$, which is in eastern part of Turkey. About $50 \mathrm{~kg}$ of mine sample was taken. Analyses showed that the sample contained $1.08 \% \mathrm{Mo}, 0.05 \% \mathrm{Cu}, 1.20 \% \mathrm{~Pb}$, $1.12 \% \mathrm{Zn}, 12.0 \% \mathrm{~F}, 0.016 \% \mathrm{Ta}, 0.20 \% \mathrm{Nb}$ and $0.14 \% \mathrm{~V}$

$98 \%(\mathrm{w} / \mathrm{v}) \mathrm{H}_{2} \mathrm{SO}_{4}, 37 \%(\mathrm{w} / \mathrm{v}) \mathrm{HCl}, 65 \%(\mathrm{w} / \mathrm{v}) \mathrm{HNO}_{3}$ and $\mathrm{KClO}_{3}$ were purchased from Merck. The K-Amyl xanthate and Aeroflot 65 used in flotation were provided from Cyanamid Co. in the USA.

A flame atomic absorption spectrometer (Unicam 929 Model AAS) was used for determination of $\mathrm{Mo}, \mathrm{Cu}, \mathrm{Pb}, \mathrm{Zn}, \mathrm{Ta}, \mathrm{Nb}$ and $\mathrm{V}$ concentrations in the solution. The fluor analysis was carried out titrimetrically [10]. Denver Mark and 890 Model pH meter were used for flotation and determination of $\mathrm{pH}$ of samples, respectively.

The sulfurization process was conducted in an autoclave of 1.3 liter internal volume, resistant to $250 \mathrm{~atm}$ pressure and to $350{ }^{\circ} \mathrm{C}$

\subsection{Method}

The mine sample was first crushed and then ground to -100 mesh size, sieved and dried at $110^{\circ} \mathrm{C}$.

For sulfurization, $1000 \mathrm{~g}$ of sample was sulfurized with gas containing different amounts $\mathrm{H}_{2} \mathrm{~S}+\mathrm{H}_{2} \mathrm{O}$ in an autoclave for 1 hour at $120^{\circ} \mathrm{C}$. Then, samples obtained from autoclave were floated with potassium amyl xanthate.

\section{RESULTS AND DISCUSSION}

\subsection{Separation of Molybdenum from Fluorite Ore:}

\subsubsection{Flotation of Original Ore}


After sieved to -100 mesh size of the original ore, flotation process was conducted under the conditions indicated blow.

Flotation condition:

$\begin{array}{ll}\text { Particle size } & :-100 \text { mesh } \\ \text { Solid / Liquid Rate } & : 100 \mathrm{~g} / \mathrm{L} \\ \text { Collector } & : 0.2 \mathrm{~g} \mathrm{Z} \text { (potassium amyl xanthate), } 3 \text { minute mix } \\ \text { Frother } & : 0,5 \mathrm{~mL} \text { Aeroflot } 65 \text { (1\%) } 2 \text { minute mix } \\ \mathrm{pH} & : 6.5-9.0 \\ \text { Mix Speed } & : 900 \mathrm{rpm}\end{array}$

At the end of flotation process, results aimed were not attained, and flotation yield was low. Approximately $12 \%$ of fluorite passed into the concentrate phase. Since an efficient separation could not be carried out, the experimental results were not given in detail. Thus, original ore was sulfurized in an autoclave before flotation. Then, these samples were floated.

\subsubsection{Flotation of Sulfurized Samples}

Firstly, samples of original fluorite ore containing molybdenum were ground, sieved to 100 mesh size, dried at $110^{\circ} \mathrm{C}$ and then reacted with gas mixtures containing different amounts of $\mathrm{H}_{2} \mathrm{~S}+\mathrm{H}_{2} \mathrm{O}$ for 1 hour at $120{ }^{\circ} \mathrm{C}$. For this purpose, the six samples each of which was $1000 \mathrm{~g}$, were reacted with mixtures of $\mathrm{H}_{2} \mathrm{~S}$ and $\mathrm{H}_{2} \mathrm{O}$ separately as seen in Table 1 .

Table 1. The used amount of gas mixtures of $\mathrm{H}_{2} \mathrm{~S}$ and $\mathrm{H}_{2} \mathrm{O}$.

\begin{tabular}{|c|c|c|}
\hline 1 & Amount of $\mathrm{H}_{2} \mathrm{~S}(\mathrm{~g})$ & Amount of $\mathrm{H}_{2} \mathrm{O}(\mathrm{g})$ \\
\hline 2 & 7.66 & 100.0 \\
3 & 11.49 & 60.0 \\
4 & 12.44 & 65.0 \\
5 & 13.40 & 70.0 \\
6 & 15.32 & 80.0 \\
\hline
\end{tabular}


After sulfurization, the samples were floated under conditions stated below.

Flotation condition:

$\begin{array}{ll}\text { Particle size } & :-100 \text { mesh Solid / Liquid Rate }: 100 \mathrm{~g} / \mathrm{L} \\ \text { Collector } & : 0.2 \mathrm{~g} \mathrm{Z}_{5} \text { (potassium amyl xanthate) } 3 \text { Minute mix } \\ \text { Frother } & : 0,5 \mathrm{~mL} \text { Aeroflot } 65(1 \%) 2 \text { Minute mix } \\ \text { Mix Speed } & : 900 \mathrm{rpm}\end{array}$

The sixth different sulfurized samples were floated under the same conditions. The results are given in Table 2-7.

Table 2: The values obtained by flotation of the first sulfurized sample

\begin{tabular}{|c|c|c|c|c|c|c|c|c|c|c|c|c|}
\hline \multirow[t]{2}{*}{$\begin{array}{l}\text { Time } \\
\text { (Min) }\end{array}$} & \multirow[t]{2}{*}{$\mathrm{pH}$} & \multirow[t]{2}{*}{$\begin{array}{l}\text { Froth } \\
\text { (g) }\end{array}$} & \multirow[t]{2}{*}{$\begin{array}{l}\text { Tailing } \\
\text { (g) }\end{array}$} & \multicolumn{3}{|c|}{$\begin{array}{l}\text { Recovery } \\
(\%)\end{array}$} & \multicolumn{3}{|c|}{$\begin{array}{c}\text { Concentrate } \\
\text { Assay (\%) }\end{array}$} & \multicolumn{3}{|c|}{$\begin{array}{l}\text { Unfloated } \\
\text { Assay (\%) }\end{array}$} \\
\hline & & & & Mo & $\mathrm{Pb}$ & $\mathrm{Cu}$ & Mo & $\mathrm{Pb}$ & $\mathrm{Cu}$ & Мo & $\mathrm{Pb}$ & $\mathrm{Cu}$ \\
\hline 3.0 & 6.50 & 33.60 & 65.50 & 96.0 & 93.6 & 53.0 & 3.05 & 3.30 & 0.08 & 0.06 & 0.11 & 0.035 \\
\hline 2.0 & 7.05 & 22.19 & 77.40 & 91.7 & 79.6 & 63.2 & 4.45 & 4.29 & 0.14 & 0.11 & 0.16 & 0.030 \\
\hline 2.3 & 7.51 & 27.95 & 71.55 & 95.0 & 83.2 & 69.2 & 3.65 & 3.57 & 0.12 & 0.08 & 0.29 & 0.020 \\
\hline 2.1 & 8.01 & 23.48 & 75.48 & 92.5 & 74.2 & 85.0 & 4.24 & 3.77 & 0.18 & 0.11 & 0.41 & 0.009 \\
\hline 1.5 & 8.53 & 22.33 & 77.16 & 95.2 & 81.0 & 77.0 & 2.60 & 4.33 & 0.17 & 0.07 & 0.30 & 0.014 \\
\hline 1.3 & 8.97 & 20.15 & 79.96 & 94.3 & 69.0 & 75.0 & 5.03 & 4.10 & 0.18 & 0.07 & 0.46 & 0.015 \\
\hline
\end{tabular}

Table 3: The values obtained by flotation of the second sulfurized sample

\begin{tabular}{|c|c|c|c|c|c|c|c|c|c|c|c|c|}
\hline \multirow[t]{2}{*}{$\begin{array}{l}\text { Time } \\
\text { (Min) }\end{array}$} & \multirow[t]{2}{*}{$\mathrm{pH}$} & \multirow[t]{2}{*}{$\begin{array}{l}\text { Froth } \\
(\mathrm{g})\end{array}$} & \multirow[t]{2}{*}{$\begin{array}{l}\text { Tailing } \\
\text { (g) }\end{array}$} & \multicolumn{3}{|c|}{$\begin{array}{l}\text { Recovery } \\
(\%)\end{array}$} & \multicolumn{3}{|c|}{$\begin{array}{c}\text { Concentrate } \\
\text { Assay (\%) }\end{array}$} & \multicolumn{3}{|c|}{$\begin{array}{l}\text { Unfloated } \\
\text { Assay (\%) }\end{array}$} \\
\hline & & & & Mo & $\mathrm{Pb}$ & $\mathrm{Cu}$ & Mo & $\mathrm{Pb}$ & $\mathrm{Cu}$ & Mo & $\mathrm{Pb}$ & $\mathrm{Cu}$ \\
\hline 1.50 & 6.54 & 29.76 & 69.56 & 94.9 & 88.6 & 67.4 & 3.42 & 3.56 & 0.11 & 0.08 & 0.19 & 0.025 \\
\hline 1.50 & 6.97 & 19.83 & 79.88 & 91.2 & 68.0 & 74.0 & 4.95 & 4.10 & 0.18 & 0.12 & 0.49 & 0.016 \\
\hline 1.15 & 7.54 & 26.59 & 72.93 & 92.3 & 78.0 & 68.5 & 3.72 & 3.50 & 0.12 & 0.11 & 0.36 & 0.022 \\
\hline 1.04 & 8.00 & 20.25 & 79.30 & 95.4 & 78.0 & 75.0 & 5.06 & 4.61 & 0.18 & 0.06 & 0.34 & 0.047 \\
\hline 1.10 & 8.52 & 20.83 & 78.38 & 91.7 & 69.4 & 65.6 & 4.73 & 3.98 & 0.15 & 0.11 & 0.47 & 0.022 \\
\hline 1.10 & 9.02 & 18.49 & 81.34 & 92.2 & 52.0 & 66.0 & 5.38 & 3.36 & 0.18 & 0.10 & 0.71 & 0.021 \\
\hline
\end{tabular}


Table 4: The values obtained by flotation of the third sulfurized sample

\begin{tabular}{|c|c|c|c|c|c|c|c|c|c|c|c|c|c|c|c|}
\hline \multirow[t]{2}{*}{$\begin{array}{l}\text { Time } \\
\text { (Min) }\end{array}$} & \multirow[t]{2}{*}{$\mathrm{pH}$} & \multirow[t]{2}{*}{$\begin{array}{l}\text { Froth } \\
\text { (g) }\end{array}$} & \multirow{2}{*}{$\begin{array}{c}\text { Tailing } \\
\text { (g) }\end{array}$} & \multicolumn{4}{|c|}{$\begin{array}{c}\text { Recovery } \\
(\%)\end{array}$} & \multicolumn{4}{|c|}{$\begin{array}{c}\text { Concentrate } \\
\text { Assay }(\%)\end{array}$} & \multicolumn{4}{|c|}{$\begin{array}{l}\text { Unfloated } \\
\text { Assay (\%) }\end{array}$} \\
\hline & & & & Mo & $\mathrm{Pb}$ & $C_{1}$ & $\mathrm{Zn}$ & & $\mathrm{Pb}$ & $\mathrm{Cu}$ & 211 & Mo & $\mathrm{Pb}$ & $\mathrm{Cu}$ & $\mathrm{Zn}$ \\
\hline 1.20 & & 26.27 & & 100 & 87.0 & 87.0 & 52.0 & 4.10 & 3.96 & 0.16 & 2.22 & & 0.21 & $\overline{0.00}$ & $\overline{0.80}$ \\
\hline 1.30 & 7.00 & 27.19 & 71.29 & 98.2 & 88.4 & 92.3 & 79.0 & 3.90 & 3.90 & 0.17 & 3.24 & 0.03 & 0.20 & 0.005 & 0.17 \\
\hline 1.30 & 7.50 & 26.74 & 72.53 & 100 & 95.9 & 95.0 & 85.8 & 4.03 & 4.29 & 0.18 & 3.58 & 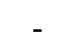 & 0.06 & 0.003 & 0.22 \\
\hline 1.30 & 8.00 & 24.80 & 74.49 & 88.7 & 79.0 & 88.9 & 43.0 & 3.86 & 3.81 & 0.18 & 1.93 & 0.16 & 0.34 & 0.007 & 0.86 \\
\hline 1.45 & 8.50 & 24.63 & 75.54 & 96.3 & 86.0 & 91.5 & 52.0 & 4.21 & 4.17 & 0.19 & 2.35 & 0.05 & 0.23 & 0.006 & 0.71 \\
\hline $1 . .30$ & 9.01 & 20.49 & 79.23 & 92.0 & 66.2 & 87.6 & 37.0 & 4.89 & 3.47 & 0.21 & 2.02 & 0.11 & 0.66 & 0.007 & 0.88 \\
\hline
\end{tabular}

Table 5: The values obtained by flotation of the fourth sulfurized sample

\begin{tabular}{|c|c|c|c|c|c|c|c|c|c|c|c|c|c|c|c|}
\hline \multirow{2}{*}{$\begin{array}{l}\text { Time } \\
\text { (Min) }\end{array}$} & \multirow[t]{2}{*}{$\mathrm{pH}$} & \multirow{2}{*}{$\begin{array}{c}\text { Froth } \\
\text { (g) }\end{array}$} & \multirow{2}{*}{$\begin{array}{c}\text { Tailing } \\
\text { (g) }\end{array}$} & \multicolumn{4}{|c|}{$\begin{array}{c}\text { Recovery } \\
(\%)\end{array}$} & \multicolumn{4}{|c|}{$\begin{array}{c}\text { Concentrate } \\
\text { Assay }(\%)\end{array}$} & \multicolumn{4}{|c|}{$\begin{array}{l}\text { Unfloated } \\
\text { Assay (\%) }\end{array}$} \\
\hline & & & & Mo & $\mathrm{Pb}$ & $\mathrm{Cu}$ & $\mathrm{Zn}$ & Mo & $\mathrm{Pb}$ & $\mathrm{Cu}$ & $\mathrm{Zn}$ & Mo & $\mathrm{Pb}$ & $\mathrm{Cu}$ & $\mathrm{Zn}$ \\
\hline 1.15 & & 831 & & 95.0 & 78.0 & 86.5 & 52.5 & 3.61 & 3.31 & 0.15 & 2.06 & 0.08 & 0.38 & 0.009 & 0.75 \\
\hline & & & & & & & & & & & & & & & \\
\hline 1.15 & 7.5 & 29.79 & 70.6 & 96.0 & 80.0 & 90. & 59.0 & 3.47 & 3.21 & 0.15 & 3. & 0.06 & 0.34 & 0.007 & 0.64 \\
\hline 1.20 & 8.00 & 27.79 & 71.7 & 99.0 & 90.0 & 88.5 & 65.0 & 3.83 & 3.87 & 0.16 & 2.60 & 0.02 & 0.17 & 0.0 & 0.55 \\
\hline 2.0 & 8.5 & 31.74 & & 94.4 & 81.0 & 86,0 & 48.0 & 3.20 & 3.05 & 0.14 & 1.69 & 0.09 & 0.34 & 0.010 & 0.86 \\
\hline 2.05 & 9.02 & 30.77 & 69.50 & 100 & 95.0 & 95.0 & 52.3 & 3.50 & 3.69 & 0.15 & 1.90 & & 0.01 & 0.003 & 0.77 \\
\hline
\end{tabular}

Table 6: The values obtained by flotation of the fifth sulfurized sample

\begin{tabular}{|c|c|c|c|c|c|c|c|c|c|c|c|c|}
\hline \multirow[t]{2}{*}{$\begin{array}{l}\text { Time } \\
\text { (Min) }\end{array}$} & \multirow[t]{2}{*}{$\mathrm{pH}$} & \multirow[t]{2}{*}{$\begin{array}{c}\text { Froth } \\
\text { (g) }\end{array}$} & \multirow{2}{*}{$\begin{array}{c}\text { Tailing } \\
\text { (g) }\end{array}$} & \multicolumn{3}{|c|}{$\begin{array}{c}\text { Recovery } \\
(\%)\end{array}$} & \multicolumn{3}{|c|}{$\begin{array}{c}\text { Concentrate } \\
\text { Assay }(\%)\end{array}$} & \multicolumn{3}{|c|}{$\begin{array}{l}\text { Unfloated } \\
\text { Assay (\%) }\end{array}$} \\
\hline & & & & Mo & $\mathrm{Pb}$ & $\mathrm{Cu}$ & Mo & $\mathrm{Pb}$ & $\mathrm{Cu}$ & Mo & $\mathrm{Pb}$ & $\mathrm{Cu}$ \\
\hline 3.0 & 6.51 & 12.04 & 86.45 & 88.5 & 72.0 & 71.0 & 7.92 & 7.16 & 0.28 & 0.15 & 0.39 & 0.017 \\
\hline 2.0 & 7.01 & 12.23 & 87.44 & 91.0 & 74.0 & 83.0 & 8.02 & 7.25 & 0.33 & 0.12 & 0.36 & 0.010 \\
\hline 2.10 & 7.51 & 12.45 & 87.11 & 90.7 & 74.4 & 81.6 & 7.85 & 7.16 & 0.32 & 0.10 & 0.35 & 0.011 \\
\hline 1.5 & 8.00 & 13.33 & 86.78 & 94.4 & 76.0 & 79.6 & 7.63 & 6.83 & 0.29 & 0.07 & 0.33 & 0.012 \\
\hline 2.1 & 8.49 & 12.21 & 87.31 & 84.0 & 78.0 & 72.0 & 7.41 & 7.65 & 0.30 & 0.20 & 0.31 & 0.016 \\
\hline 2.36 & 9.0 & 13.69 & 86.28 & 89.8 & 81.0 & 74.8 & 6.79 & 7.09 & 0.26 & 0.13 & 0.25 & 0.014 \\
\hline
\end{tabular}


Table 7: The values obtained by flotation of the sixth sulfurized sample

\begin{tabular}{|c|c|c|c|c|c|c|c|c|c|c|c|c|}
\hline \multirow{2}{*}{$\begin{array}{l}\text { Time } \\
\text { (Min) }\end{array}$} & \multirow[t]{2}{*}{$\mathrm{pH}$} & \multirow{2}{*}{$\begin{array}{c}\text { Froth } \\
\text { (g) }\end{array}$} & \multirow{2}{*}{$\begin{array}{c}\text { Tailing } \\
\text { (g) }\end{array}$} & \multicolumn{3}{|c|}{$\begin{array}{c}\text { Recovery } \\
(\%)\end{array}$} & \multicolumn{3}{|c|}{$\begin{array}{c}\text { Concentrate } \\
\text { Assay }(\%)\end{array}$} & \multicolumn{3}{|c|}{$\begin{array}{l}\text { Unfloated } \\
\text { Assay (\%) }\end{array}$} \\
\hline & & & & Mo & $\mathrm{Pb}$ & $\mathrm{Cu}$ & Mo & $\mathrm{Pb}$ & $\mathrm{Cu}$ & Mo & $\mathrm{Pb}$ & $\mathrm{Cu}$ \\
\hline 2.0 & 7.00 & 29.21 & 69.69 & 89.3 & 73,0 & 80.0 & 3.29 & 2.98 & 0.13 & 0.16 & 0.46 & 0.014 \\
\hline 4.0 & 7.02 & 33.33 & 215.34 & 91.4 & 82.1 & 88.4 & 7.40 & 7.37 & 0.33 & 0.10 & 0.25 & 0.007 \\
\hline 5.0 & 7.00 & 70.40 & 228.43 & 89.8 & 91.7 & 83.5 & 4.13 & 4.68 & 0.17 & 0.15 & 0.13 & 0.011 \\
\hline 7.2 & 7.01 & 90.66 & 257.22 & 90.6 & 95.0 & 85.7 & 3.78 & 4.40 & 0.16 & 0.14 & 0.08 & 0.010 \\
\hline
\end{tabular}

As seen in tables, the best result was obtained from third sulfurized sample by flotation. In order to understand these results better, the results were plotted in Figure 1 with respect to different $\mathrm{pH}$ values.

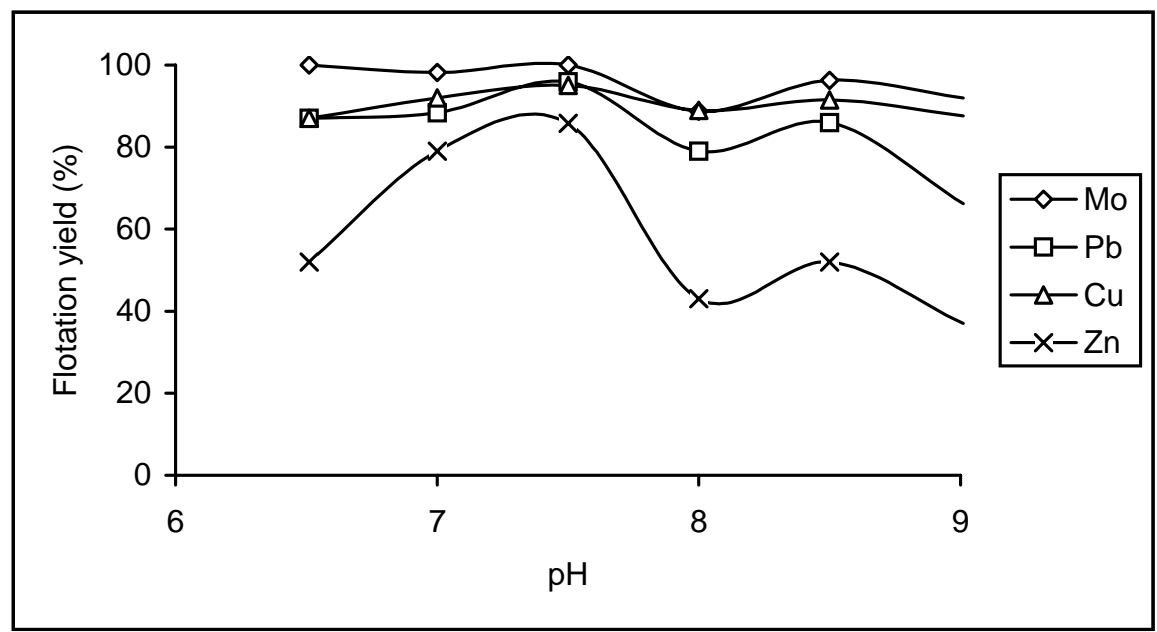

Figure 1: The effect of $\mathrm{pH}$ on the flotation yield

As seen in Figure 1, the best of flotation yield was at $\mathrm{pH}$ 7.5. Thus, six different sulfurized samples were floated at this $\mathrm{pH}$ and the results are given in Figure 2. 


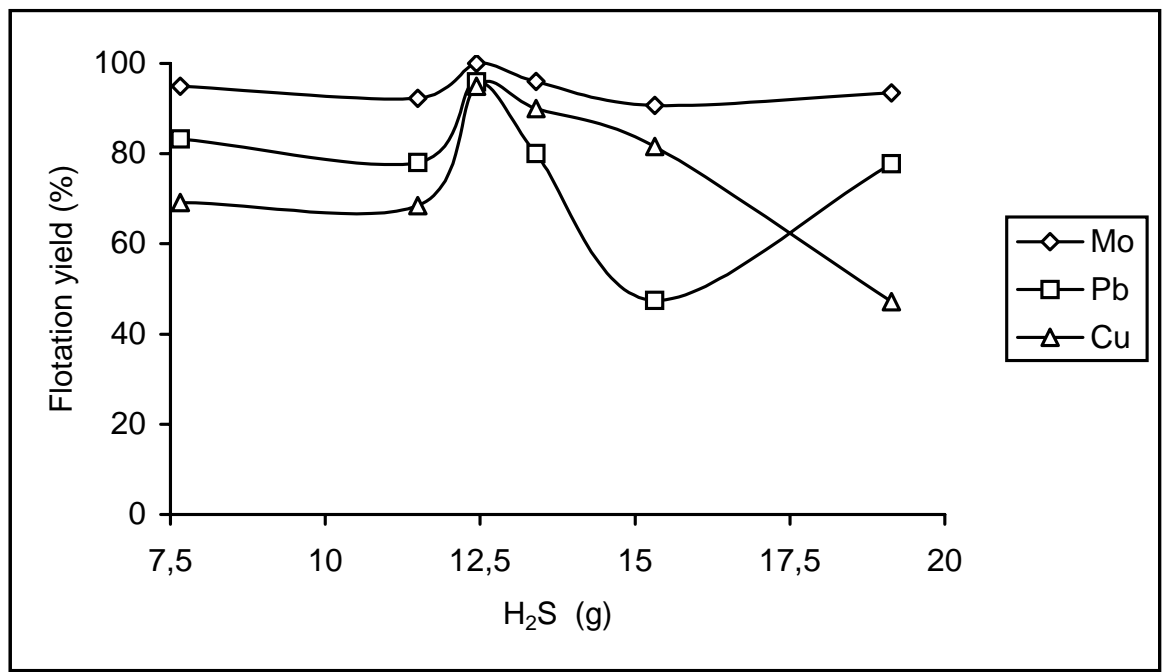

Figure 2: The effect of sulfurization at $\mathrm{pH} 7.5$ on the flotation yield of sulfurized samples

When Figure 2 is examined, it can be seen that the highest flotation yield was achieved by flotation of third sulfurized samples.

In the next step, flotation process was conducted to test the effect of pulp density and results are given in Table 8 .

Table 8: The effect of pulp density on the floatability of results from third sulfurized sample

\begin{tabular}{|c|c|c|c|c|c|c|c|c|c|c|c|c|c|c|c|}
\hline \multirow[t]{2}{*}{$\begin{array}{l}\text { Time } \\
\text { (Min) }\end{array}$} & \multirow[t]{2}{*}{$\mathrm{pH}$} & \multirow[t]{2}{*}{$\begin{array}{l}\text { Froth } \\
\text { (g) }\end{array}$} & \multirow{2}{*}{$\begin{array}{c}\text { Tailing } \\
\text { (g) }\end{array}$} & \multicolumn{4}{|c|}{$\begin{array}{c}\text { Recovery } \\
(\%)\end{array}$} & \multicolumn{4}{|c|}{$\begin{array}{c}\text { Concentrate } \\
\text { Assay }(\%)\end{array}$} & \multicolumn{4}{|c|}{$\begin{array}{l}\text { Unfloated } \\
\text { Assay (\%) }\end{array}$} \\
\hline & & & & Mo & $\mathrm{Pb}$ & $\mathrm{Cu}$ & $\mathrm{Zn}$ & Mo & $\mathrm{Pb}$ & $\mathrm{Cu}$ & $\mathrm{Zn}$ & Mo & $\mathrm{Pb}$ & $\mathrm{Cu}$ & $\mathrm{Zn}$ \\
\hline 2.40 & 7.50 & 25.73 & 73.52 & 97.4 & 94.6 & 94.0 & 86.0 & 4.08 & 4.41 & 0.18 & 3.74 & 0.04 & 0.06 & 0.004 & 0.21 \\
\hline 3.50 & 7.51 & 61.84 & 188.73 & 96.4 & 93.0 & 94.0 & 85.5 & 4.20 & 4.50 & 0.19 & 3.86 & 0.05 & 0.11 & 0.003 & 0.22 \\
\hline 3.60 & 7.52 & 67.04 & 232.23 & 97.6 & 94.0 & .95 .4 & 89.0 & 4.71 & 5.03 & 0.21 & 4.45 & 0.03 & 0.09 & 0.004 & 0.16 \\
\hline 3.75 & 7.50 & 83.33 & 266.20 & 94.6 & 92.0 & 85.6 & 82.0 & 4.29 & 4.62 & 0.17 & 3.86 & 0.08 & 0.13 & 0.003 & 0.27 \\
\hline
\end{tabular}

When Table 8 is examined, it can be seen that there is not a significant difference in flotation yield as pulp density changes. However, the results are better when solid/liquid ratio was done at $300 \mathrm{~g}$ ore $/ 1 \mathrm{~L}$

\subsubsection{The effect of the activator and the depressant on the flotation yield}

\subsubsection{The effect of the activator:}


The results of the flotation in which $\mathrm{CuSO}_{4}$ was used as activator are given in Table 9.

Table 9: The values obtained from flotation of third sulfurized sample using activator $\left(\mathrm{CuSO}_{4}\right)$

\begin{tabular}{|c|c|c|c|c|c|c|c|c|c|c|c|c|c|c|c|c|}
\hline \multirow[t]{2}{*}{$\begin{array}{l}\mathrm{CuSO}_{4} \\
(\mathrm{mg})\end{array}$} & \multirow[t]{2}{*}{$\begin{array}{l}\text { Time } \\
\text { Min }\end{array}$} & \multirow[t]{2}{*}{$\mathrm{pH}$} & \multirow[t]{2}{*}{$\begin{array}{c}\text { Froth } \\
\text { (g) }\end{array}$} & \multirow{2}{*}{$\begin{array}{l}\text { Tail } \\
\text { (g) }\end{array}$} & \multicolumn{4}{|c|}{$\begin{array}{c}\text { Recovery } \\
(\%)\end{array}$} & \multicolumn{4}{|c|}{$\begin{array}{c}\text { Concentrate } \\
\text { Assay }(\%)\end{array}$} & \multicolumn{4}{|c|}{$\begin{array}{l}\text { Unfloated } \\
\text { Assay (\%) }\end{array}$} \\
\hline & & & & & Mo & $\mathrm{Pb}$ & $\mathrm{Cu}$ & $\mathrm{Zn}$ & Mo & $\mathrm{Pb}$ & $\mathrm{Cu}$ & $\mathrm{Zn}$ & Mo & $\mathrm{Pb}$ & $\mathrm{Cu}$ & $\mathrm{Zn}$ \\
\hline 5.0 & 1.50 & 7.50 & 33.00 & 66.32 & 100 & 87.0 & 80.0 & 60.0 & 3.27 & 3.16 & 0.12 & 2.03 & - & 0.24 & 0.015 & 0.68 \\
\hline 10.0 & 1.20 & 7.52 & 38.83 & 59.92 & 100 & 84.0 & 99.0 & 92.0 & 2.78 & 2.59 & 0.12 & 2.65 & - & 0.33 & 0.001 & 0.15 \\
\hline 10.0 & 1.20 & 8.50 & 31.83 & 67.98 & 100 & 90.0 & 95.0 & 72.0 & 3.05 & 3.38 & 0.15 & 2.52 & - & 0.18 & 0.004 & 0.46 \\
\hline
\end{tabular}

As seen in Table 9, floated amount of sample was too much. Moreover, in order to investigate whether flotation yield could be increase more, a series of experiments were carried out by using depressant $\left(\mathrm{Na}_{2} \mathrm{SiO}_{3}\right)$ as well as activator.

\subsubsection{The effect of the activator and depressant together:}

In these series of experiments, flotation studies were conducted by using $\mathrm{CuSO}_{4}$ and $\mathrm{Na}_{2} \mathrm{SiO}_{3}$ and results obtained are given in Table 10 .

Table 10: The values obtained from flotation of third sulfurized sample using the activator and the depressant together at $\mathrm{pH} 7.5$

\begin{tabular}{|c|c|c|c|c|c|c|c|c|c|c|c|c|c|c|c|c|}
\hline \multirow[t]{2}{*}{$\begin{array}{c}\mathrm{CuSO}_{4} \\
(\mathrm{mg})\end{array}$} & \multirow{2}{*}{$\begin{array}{c}\mathrm{Na}_{2} \mathrm{Si} \\
\mathrm{O}_{3} \\
(\mathrm{~mL})\end{array}$} & \multirow[t]{2}{*}{$\begin{array}{l}\text { Time } \\
\text { (Min) }\end{array}$} & \multirow[t]{2}{*}{$\begin{array}{l}\text { Froth } \\
(\mathrm{g})\end{array}$} & \multirow[t]{2}{*}{$\begin{array}{l}\text { Tail } \\
\text { (g) }\end{array}$} & \multicolumn{4}{|c|}{$\begin{array}{c}\text { Recovery } \\
(\%)\end{array}$} & \multicolumn{4}{|c|}{$\begin{array}{c}\text { Concentrate } \\
\text { Assay }(\%)\end{array}$} & \multicolumn{4}{|c|}{$\begin{array}{l}\text { Unfloated } \\
\text { Assay (\%) }\end{array}$} \\
\hline & & & & & Mo & $\mathrm{Pb}$ & $\mathrm{Cu}$ & $\mathrm{Zn}$ & Mo & $\mathrm{Pb}$ & $\mathrm{Cu}$ & $\mathrm{Zn}$ & Mo & $\mathrm{Pb}$ & $\mathrm{Cu}$ & $\mathrm{Zn}$ \\
\hline 5.0 & 0.5 & 1.30 & 29.85 & 70.29 & 100 & 87.0 & 94.0 & 66.0 & 3.61 & 3.48 & 0.15 & 2.46 & - & 0.23 & 0.004 & 0.55 \\
\hline 15.0 & 1.0 & 2.10 & 19.75 & 79.66 & 95.0 & 80.0 & 84.0 & 78.0 & 5.18 & 4.85 & 0.21 & 4.41 & 0.07 & 0.31 & 0.010 & 0.31 \\
\hline 20.0 & 1.50 & 2.20 & 19.56 & 79.91 & 91.0 & 66.0 & 55.0 & 67.0 & 5.01 & 4.03 & 0.14 & 3.82 & 0.13 & 0.52 & 0.028 & 0.52 \\
\hline 20.0 & 1.0 & 2.30 & 19.94 & 79.43 & 96.0 & 67.0 & 76.0 & 69.0 & 5.18 & 4.02 & 0.19 & 3.86 & 0.05 & 0.50 & 0.015 & 0.44 \\
\hline 20.0 & 0.5 & 2.20 & 22.74 & 78.27 & 89.0 & 73.0 & 72.0 & 65.0 & 4.21 & 3.84 & 0.15 & 3.20 & 0.15 & 0.42 & 0.018 & 0.51 \\
\hline 25.0 & 1.0 & 1.50 & 24.90 & 74.64 & 96.3 & 92.0 & 95.6 & 89.0 & 4.16 & 4.42 & 0.19 & 4.00 & 0.05 & 0.13 & 0.003 & 0.17 \\
\hline $30.0 *$ & 1.0 & 1.40 & 23.47 & 75.92 & 97.3 & 93.0 & 98.0 & 95.2 & 4.46 & 4.74 & 0.21 & 4.53 & 0.04 & 0.11 & 0.001 & 0.07 \\
\hline 35.0 & 1.0 & 1.30 & 23.85 & 75.49 & 96.3 & 86.0 & 100 & 66.2 & 4.35 & 4.31 & 0.21 & 3.10 & 0.05 & 0.22 & - & 0.50 \\
\hline
\end{tabular}

Note: $1 \mathrm{~mL}$ solution contains $5.10^{-3} \mathrm{~g} \mathrm{Na}_{2} \mathrm{SiO}_{3}$.

* Conditions under which the best results were obtained for $\mathrm{Mo}, \mathrm{Cu}, \mathrm{Pb}$ and $\mathrm{Zn}$ recovery 
As seen in Table 10, although molybdenum yield decreased a bit, yields of other elements increased considerably. Particularly, the optimum results were obtained when $30.0 \mathrm{mg} \mathrm{CuSO}_{4}$ and $1.0 \mathrm{~mL} \mathrm{Na} 2 \mathrm{SiO}_{3}$ were used in the flotation. In addition, amount of sample floated by depressant fairly decreased and $94 \%$ of fluorite was depressed (not shown in Table). The results obtained from flotation of the samples at up to $30 \%$ solid/liquid ratio as the pulp density changed were similar (not shown in Table). Additionally, this concentrate phase (flotation carried out with $30 \mathrm{mg}$ $\mathrm{CuSO}_{4}$ ) contained $0.05 \% \mathrm{Ta}, 0.68 \% \mathrm{Nb}$ and $0.41 \% \mathrm{~V}$ and was produced with recoveries of $76 \%$ $\mathrm{Ta}, 84.4 \% \mathrm{Nb}$ and $76.2 \% \mathrm{~V}$ by flotation.

\section{Separation of Mo and $\mathrm{Cu}$ from $\mathrm{Pb}$ and $\mathrm{Zn}$ in the Concentrate}

The concentrate phase (flotation carried out with $30 \mathrm{mg} \mathrm{CuSO}$ ) containing $4.46 \% \mathrm{Mo}$, $4.74 \% \mathrm{~Pb}, 0.21 \% \mathrm{Cu}, 4.53 \% \mathrm{Zn}, 0.05 \% \mathrm{Ta}, 0.68 \% \mathrm{Nb}$ and $0.41 \% \mathrm{~V}$ was used in this process. In order to separate $\mathrm{Mo}$ and $\mathrm{Cu}$ from $\mathrm{Pb}$ and $\mathrm{Zn}$, the experiments were conducted with respect to $\mathrm{pH}$. Thus, these experiments were performed with -100 mesh size and -160 mesh sizes, and $\mathrm{pH} 11$ to $\mathrm{pH}$ 13. Results obtained are given in Table 11.

Table 11: The values obtained by flotation of the collective concentrate

\begin{tabular}{|c|c|c|c|c|c|c|c|c|c|c|c|c|c|c|c|}
\hline \multirow{3}{*}{$\begin{array}{l}\text { Time } \\
\text { (Min) }\end{array}$} & \multirow[t]{3}{*}{$\mathrm{pH}$} & \multirow{3}{*}{$\begin{array}{l}\text { Froth } \\
(\mathrm{g})\end{array}$} & \multirow{3}{*}{$\begin{array}{l}\text { Tail } \\
\text { (g) }\end{array}$} & \multicolumn{12}{|c|}{-100 mesh size } \\
\hline & & & & \multicolumn{4}{|c|}{$\begin{array}{c}\text { Recovery } \\
(\%)\end{array}$} & \multicolumn{4}{|c|}{$\begin{array}{c}\text { Concentrate } \\
\text { Assay }(\%)\end{array}$} & \multicolumn{4}{|c|}{$\begin{array}{l}\text { Unfloated } \\
\text { Assay (\%) }\end{array}$} \\
\hline & & & & Mo & $\mathrm{Pb}$ & $\mathrm{Cu}$ & $\mathrm{Zn}$ & Mo & $\mathrm{Pb}$ & $\mathrm{Cu}$ & $\mathrm{Zn}$ & Mo & $\mathrm{Pb}$ & $\mathrm{Cu}$ & $\mathrm{Zn}$ \\
\hline 2.50 & 11.0 & 78.20 & 21.55 & 98.0 & 97.6 & 98.0 & 87.7 & 5.58 & 5.90 & 0.26 & 5.07 & 0.42 & 0.53 & 0.019 & 2.59 \\
\hline 2.30 & 11.50 & 62.34 & 37.71 & 95.5 & 94.0 & 96.7 & 75.8 & 6.82 & 7.14 & 0.32 & 5.50 & 0.53 & 0.75 & 0.018 & 2.90 \\
\hline 2.50 & 12.00 & 50.79 & 48.97 & 90.0 & 77.6 & 93.9 & 60.5 & 7.90 & 7.23 & 0.38 & 5.39 & 0.91 & 3.14 & 0.026 & 3.65 \\
\hline 2.50 & 12.50 & 45.26 & 53.43 & 98.0 & 57.0 & 65.8 & 55.0 & 9.65 & 5.96 & 0.30 & 5.49 & 0.17 & 3.82 & 0.134 & 3.82 \\
\hline 2.50 & 12.75 & 38.59 & 61.04 & 93.0 & 47.5 & 74.0 & 25.4 & 10.74 & 5.82 & 0.40 & 2.98 & 0.51 & 4.08 & 0.089 & 5.54 \\
\hline 3.00 & 13.00 & 31.31 & 69.87 & 97.3 & 31.6 & 84.0 & 30.0 & 13.85 & 4.77 & 0.56 & 4.33 & 0.17 & 4.64 & 0.048 & 4.54 \\
\hline & & & & \multicolumn{12}{|c|}{-160 mesh size } \\
\hline 3.00 & 13.0 & 33.46 & 66.98 & 84.6 & 31.3 & 68.4 & 34.4 & 11.27 & 4.41 & 0.43 & 4.65 & 1.03 & 4.86 & 0.124 & 4.97 \\
\hline
\end{tabular}

As seen in Table 11, the best separation was performed at $\mathrm{pH} 13$ and with -100 mesh particle size. However, in order to obtain better flotation results, -100 mesh size samples were sulfurized again. For resulfurization, two samples were prepared in two different autoclave mediums: Sample A containing $0.96 \mathrm{~g} \mathrm{H}_{2} \mathrm{~S}+5 \mathrm{~g} \mathrm{H}_{2} \mathrm{O}$ steam for $500 \mathrm{~g}$ of sample and Sample B containing $1.92 \mathrm{~g} \mathrm{H}_{2} \mathrm{~S}+10 \mathrm{~g} \mathrm{H}_{2} \mathrm{O}$ for $500 \mathrm{~g}$ of sample. Additionally, -200 mesh size samples were 
sulfurized under the same conditions. The best results were obtained from the flotation of the samples of -100 mesh size sulfurized in a medium containing $0.96 \mathrm{~g} \mathrm{H}_{2} \mathrm{~S}+5 \mathrm{~g} \mathrm{H}_{2} \mathrm{O}$ steam for 500 $\mathrm{g}$ of sample and are given in Table 12.

Table 12: The values obtained by flotation of resulfurized collective concentrate

\begin{tabular}{|c|c|c|c|c|c|c|c|c|c|c|c|c|c|c|c|}
\hline \multirow[t]{2}{*}{$\begin{array}{l}\text { Time } \\
\text { (Min) }\end{array}$} & \multirow[t]{2}{*}{$\mathrm{pH}$} & \multirow[t]{2}{*}{$\begin{array}{l}\text { Froth } \\
(\mathrm{g})\end{array}$} & \multirow{2}{*}{$\begin{array}{l}\text { Tailing } \\
\text { (g) }\end{array}$} & \multicolumn{4}{|c|}{$\begin{array}{c}\text { Recovery } \\
(\%)\end{array}$} & \multicolumn{4}{|c|}{$\begin{array}{c}\text { Concentrate } \\
\text { Assay (\%) }\end{array}$} & \multicolumn{4}{|c|}{$\begin{array}{l}\text { Unfloated } \\
\text { Assay (\%) }\end{array}$} \\
\hline & & & & Mo & $\mathrm{Pb}$ & $\mathrm{Cu}$ & $\mathrm{Zn}$ & Mo & $\mathrm{Pb}$ & $\mathrm{Cu}$ & $\mathrm{Zn}$ & Mo & $\mathrm{Pb}$ & $\mathrm{Cu}$ & $\mathrm{Zn}$ \\
\hline 3.0 & 12.00 & 74.06 & 25.88 & 97.6 & 94.0 & 97.0 & 83.0 & 5.87 & 6.01 & 0.27 & 5.06 & 0.41 & 1.10 & 0.024 & 2.98 \\
\hline 2.5 & 12.50 & 36.05 & 64.68 & 96.0 & 64.0 & 88.0 & 44.0 & 11.87 & 8.40 & 0.51 & 5.51 & 0.28 & 2.64 & 0.038 & 3.92 \\
\hline 2.30 & 13.00 & 22.45 & 77.69 & 98.0 & 12.6 & 94.0 & 22.5 & 19.46 & 2.65 & 0.87 & 4.54 & 0.11 & 5.33 & 0.016 & 4.52 \\
\hline
\end{tabular}

As seen in Table 12, most of $\mathrm{Mo}$ and $\mathrm{Cu}$ were floated at $\mathrm{pH} 13$, while most of $\mathrm{Pb}$ and $\mathrm{Zn}$ were depressed. Then, $\mathrm{Mo}, \mathrm{Cu}, \mathrm{Ta}, \mathrm{Nb}$ and $\mathrm{V}$ were recovered from this concentrate. For this process, these elements were separated by a hydrometallurgical method (9).

Moreover, the flotation yields of $\mathrm{Ta}, \mathrm{Nb}$ and $\mathrm{V}$, which were not given in Table 12, were found as $86 \%, 91 \%$ and $89 \%$, respectively and their concentrate grades of $\mathrm{Ta}, \mathrm{Nb}$ and $\mathrm{V}$ were ascertained as $0.16 \% \mathrm{Ta}, 2.43 \% \mathrm{Nb}$ and $1.49 \% \mathrm{~V}$.

In the next step, the sample was floated to test the effects of pulp density. As results given in Table 12, the best of yield was obtained with $10 \%$ solid/liquid ratio.

\section{Flotation of Fluorite in the Tailing}

Tailing of first step flotation (collective flotation) contained $14.4 \% \mathrm{~F}\left(29.6 \% \mathrm{CaF}_{2}\right)$. It was tried to enrich fluorite by flotation of this sample. For this aim, the effect of some parameters such as $\mathrm{pH}$, amount of the collector, the activator and the depressant were examined. Results obtained under flotation conditions stated below are given in Table 13.

Flotation condition:

$$
\begin{array}{ll}
\text { Particle size } & :-100 \text { mesh } \\
\text { Solid / Liquid Rate } & : 100 \mathrm{~g} / \mathrm{L} \\
\text { Collector } & : \text { Na-Oleate }\left(5.10^{-2} \mathrm{~g} / \mathrm{mL}\right) 3 \text { minute mix }
\end{array}
$$


Activator

Suppressor

Mix Speed
$: \mathrm{Al}_{2}\left(\mathrm{SO}_{4}\right)_{3}\left(5.10^{-2} \mathrm{~g} / \mathrm{mL}\right)$

$: \mathrm{Na}_{2} \mathrm{SiO}_{3}\left(5.10^{-3} \mathrm{~g} / \mathrm{mL}\right)$

: $900 \mathrm{rpm}$

Table 13: The values obtained from the flotation performed for enrichment of fluorite

\begin{tabular}{|c|c|c|c|c|c|c|c|c|c|c|c|}
\hline \multirow[t]{2}{*}{$\begin{array}{l}\text { Time } \\
\text { (Min) }\end{array}$} & \multirow[t]{2}{*}{$\begin{array}{l}\mathrm{Al}_{2}\left(\mathrm{SO}_{4}\right)_{3} \\
\quad(\mathrm{~mL})\end{array}$} & \multirow[t]{2}{*}{$\begin{array}{l}\mathrm{Na}_{2} \mathrm{SiO}_{3} \\
(\mathrm{~mL})\end{array}$} & \multirow{2}{*}{$\begin{array}{c}\mathrm{Na} \\
\text { oleat } \\
(\mathrm{mL})\end{array}$} & \multirow[t]{2}{*}{$\mathrm{pH}$} & \multirow{2}{*}{$\begin{array}{l}\text { Froth } \\
\text { (g) }\end{array}$} & \multirow{2}{*}{$\begin{array}{c}\text { Tailing } \\
\text { (g) }\end{array}$} & \multirow{2}{*}{$\begin{array}{c}\begin{array}{c}\text { Recovery } \\
(\%)\end{array} \\
\mathrm{F}\end{array}$} & \multicolumn{2}{|c|}{$\begin{array}{c}\text { Concentrate } \\
\text { Assay }(\%)\end{array}$} & \multicolumn{2}{|c|}{$\begin{array}{l}\text { Unfloated } \\
\text { Assay (\%) }\end{array}$} \\
\hline & & & & & & & & $F$ & $\mathrm{CaF}_{2}$ & $F$ & $\mathrm{CaF}_{2}$ \\
\hline 1.0 & 1.0 & 1.0 & 1.0 & 7.5 & 24.77 & 74.49 & 78.0 & 45.35 & 93.21 & 4.2 & 8.7 \\
\hline 1.0 & 1.0 & 1.0 & 1.0 & 8.0 & 16.81 & 82.48 & 44.4 & 38.03 & 78.18 & 9.7 & 19.9 \\
\hline 1.10 & 1.0 & 1.0 & 1.0 & 8.5 & 19.20 & 80.53 & 53.2 & 39.90 & 82.02 & 7.3 & 15.7 \\
\hline 1.20 & 1.0 & 1.0 & 1.0 & 9.0 & 24.39 & 75.03 & 71.3 & 42.10 & 86.53 & 5.5 & 11.3 \\
\hline
\end{tabular}

$* 1.0 \mathrm{~mL} \mathrm{Al}_{2}\left(\mathrm{SO}_{4}\right)_{3}=5.10^{-2} \mathrm{~g}, 1.0 \mathrm{~mL} \mathrm{Na}_{2} \mathrm{SiO}_{3}=5.10^{-3} \mathrm{~g}, 1.0 \mathrm{~mL}$ Na-Oleate $=5.10^{-2} \mathrm{~g}$

As seen in Table 13, the highest flotation yield was at $\mathrm{pH}$ 7.5. However, since these results were not satisfactory, the sample was floated with respect to the amounts of the activator, the depressant and the collector and the results obtained are given in Table 14.

Table 14: The values obtained from the flotation performed with respect to the activator, depressant and collector

\begin{tabular}{|c|c|c|c|c|c|c|c|c|c|}
\hline \multirow{2}{*}{$\begin{array}{c}\mathrm{Al}_{2}\left(\mathrm{SO}_{4}\right)_{3} \\
\mathrm{Na}_{2} \mathrm{SiO}_{3} \\
\mathrm{Na} \text { oleate } \\
(\mathrm{mL})\end{array}$} & \multirow[t]{2}{*}{$\begin{array}{l}\text { Time } \\
\text { (Min) }\end{array}$} & \multirow[t]{2}{*}{$\mathrm{pH}$} & \multirow{2}{*}{$\begin{array}{c}\text { Froth } \\
(\mathrm{g})\end{array}$} & \multirow{2}{*}{$\begin{array}{c}\text { Tailing } \\
\text { (g) }\end{array}$} & \multirow{2}{*}{$\begin{array}{c}\text { Recovery } \\
(\%)\end{array}$} & \multicolumn{2}{|c|}{$\begin{array}{c}\text { Concentrate } \\
\text { Assay (\%) }\end{array}$} & \multicolumn{2}{|c|}{$\begin{array}{l}\text { Unfloated } \\
\text { Assay (\%) }\end{array}$} \\
\hline & & & & & & $\mathrm{F}$ & $\mathrm{CaF}_{2}$ & $\mathrm{~F}$ & $\mathrm{CaF}_{2}$ \\
\hline $\begin{array}{c}- \\
1.0 \\
1.0\end{array}$ & 1.50 & 7.5 & 29.03 & 70.40 & 94.0 & 46.63 & 95.85 & 1.2 & 2.5 \\
\hline $\begin{array}{l}1.0 \\
1.0 \\
2.0\end{array}$ & 2.0 & 7.50 & 32.39 & 66.48 & 95.0 & 42.24 & 86.82 & 1.1 & 2.2 \\
\hline $\begin{array}{l}1.0 \\
1.0 \\
1.5\end{array}$ & 1.5 & 8.0 & 15.77 & 83.57 & 48.0 & 43.83 & 90.10 & 8.9 & 18.4 \\
\hline $\begin{array}{l}1.0 \\
1.0 \\
2.0 \\
\end{array}$ & 2.0 & 8.0 & 35.61 & 63.76 & 94.5 & 38.21 & 78.55 & 1.2 & 2.6 \\
\hline $\begin{array}{l}1.0 \\
0.5 \\
1.0\end{array}$ & 1.45 & 7.5 & 23.51 & 76.90 & 73.4 & 44.96 & 92.41 & 5.0 & 10.2 \\
\hline $\begin{array}{l}1.0 \\
0.5 \\
1.0\end{array}$ & 2.0 & 8.0 & 30.23 & 68.96 & 94.0 & 44.78 & 92.04 & 1.3 & 2.6 \\
\hline
\end{tabular}


As seen in Table 14, the highest flotation yield and in concentrate grade of $\mathrm{CaF}_{2}$ were obtained by the flotation conducted in a medium containing $1.0 \mathrm{~mL} \mathrm{Na} \mathrm{SiO}_{3}$ and $1.0 \mathrm{~mL} \mathrm{Na-}$ oleate. Thus, these experiments were repeated three times and the similar results were obtained. Then, the flotation studies were performed by changing the amounts of $\mathrm{Na}_{2} \mathrm{SiO}_{3}$ and $\mathrm{Na}$ - oleate. The best result was obtained from the flotation in which $1.0 \mathrm{~mL} \mathrm{Na} \mathrm{SiO}_{3}$ and $1.0 \mathrm{~mL} \mathrm{Na-} \mathrm{oleate}$ was used at $\mathrm{pH} 7.5$ and it was determined that grade of sulfur was $0.5 \%$.

\section{CONCLUSIONS}

The finding of processing of fluorite ore containing molybdenum are as follows.

- Collecting molybdenum and fluorite in different phases by direct flotation was not possible, and the flotation yield was low

- Since molybdenum and other minerals bound to gang minerals, phase separation was not possible. Liberation with respect to particle size was unsatisfactory and this resulted in low flotation yield.

- In order to achieve an efficient separation by flotation, fluorite ore containing molybdenum was sulfurized to change its structure and surface. Thus, the sample was sulfurized in a medium containing different amounts of $\mathrm{H}_{2} \mathrm{~S}$ and $\mathrm{H}_{2} \mathrm{O}$ steam. $12.44 \mathrm{~g} \mathrm{H}_{2} \mathrm{~S}+65 \mathrm{~g} \mathrm{H}_{2} \mathrm{O}$ gas mixture was enough for sulfurization one $\mathrm{kg}$ of sieved ore. The sulfurization was finished in 1 hour in the autoclave for at $120^{\circ} \mathrm{C}$.

- The minerals containing fluorite and molybdenum were separated with high yields by flotation of sulfurized sample. At the end of procedure, $94 \%$ of fluorite was remained at the tailing. Beside of Molybdenum, $\mathrm{Pb}, \mathrm{Zn}, \mathrm{Cu}, \mathrm{Ta}, \mathrm{Nb}$ and $\mathrm{V}$ also passed into concentrate phase with high yields. After the collective flotation, concentrate phase contained $4.46 \% \mathrm{Mo}, 4.74 \% \mathrm{~Pb}, 4.53 \% \mathrm{Zn}, 0.21 \% \mathrm{Cu}, 0.05 \%$ $\mathrm{Ta}, 0.68 \% \mathrm{Nb}$ and $0.41 \% \mathrm{~V}$. In the flotation performed with respect to pulp density, the flotation yield was remained at the same levels with the samples consisted of up to $30 \%$ solid/liquid ratio. Additionally, the flotation yield was increased when $\mathrm{Na}_{2} \mathrm{SiO}_{3}$ was used as depressant and $\mathrm{CuSO}_{4}$ was used as activator.

- In order to increase the grade of $\mathrm{Mo}, \mathrm{Cu}, \mathrm{Ta}, \mathrm{Nb}$ and $\mathrm{V}$ from $\mathrm{Zn}$ and $\mathrm{Pb}$, the flotation process was repeated. Flotation was performed at higher $\mathrm{pH}$ and optimum $\mathrm{pH}$ was found as 13 . However, it was decided to repeat sulfurization of concentrate since flotation yield was not satisfactory. 
- $0.96 \mathrm{~g} \mathrm{H}_{2} \mathrm{~S}+5 \mathrm{~g} \mathrm{H}_{2} \mathrm{O}$ gas mixtures were enough for the sulfurization of $500 \mathrm{~g}$ sample. After the flotation, the concentrate grades of $\mathrm{Mo}, \mathrm{Pb}, \mathrm{Cu}, \mathrm{Zn}, \mathrm{Ta}, \mathrm{Nb}$ and $\mathrm{V}$ were found as $19.46 \%, 2.65 \%, 0.87 \%, 4.54 \%, 0.16 \%, 2.43 \%$, and $1.49 \%$ and flotation yields were determined as $98 \%, 12.6 \%, 94 \%, 22.5 \%, 86 \%, 94 \%$ and $82.2 \%$, respectively. So, while $\mathrm{Mo}, \mathrm{Cu}, \mathrm{Ta}, \mathrm{Nb}$ and $\mathrm{V}$ was collected in the concentrate phase, $\mathrm{Pb}$ and $\mathrm{Zn}$ passed into the tailing. The concentrate was enriched approximately 10 times with respect to the original ore.

- It was ascertained that the tailing of collective flotation contained $14.4 \% \mathrm{~F}(29.6 \%$ $\mathrm{CaF}_{2}$ ). This sample was refloated for enrichment. The effect of the parameters such as $\mathrm{pH}$, activator, depressant and collector were investigated. The highest flotation yield was at $\mathrm{pH}$ 7.5. Using $\mathrm{Al}_{2}\left(\mathrm{SO}_{4}\right)_{3}$ as activator did not increased the flotation yield significantly. On the contrary, in the flotation in which $\mathrm{Na}_{2} \mathrm{SiO}_{3}$ and $\mathrm{Na}$ oleate was used, concentrate grade of $\mathrm{CaF}_{2}$ raised to $96 \%$ and flotation yield was $94 \%$. Additionally, it was determined that the concentrate contained $0.5 \% \mathrm{~S}$.

\section{REFERENCES}

1. De Leeuw, N.H., Parker, S.C., Rao, K.H., Modeling the competitive adsorption of water and methanoic acid on calcite and fluorite surfaces. Langmuir 14, 5900-5906 (1998).

2. Helbig, C., Baldauf, H., Mahnke, J., Stochelhuber, K.W., Schulze, H.J., Investigation of Langmuir monofilms and flotation experiments with anionic/cationic collector mixtures. International Journal of Mineral Processing 53, 135-144 (1998).

3. Helbig, C., Baldauf, H., Lange, T., Newmann, R., Pollex, R., Weber, E., New binary collectors with increased otation Tenside Surfactants Detergents 36, 58-62 (1999).

4. Zhou, Q., Lu, S., Acidized sodium silicate an effective modifier in .fluorite flotation, Minerals Engineering 5, 435-444 (1992).

5. MTA Report no 76, project no 80-45, Turkey (1981)

6. Qiang Zhou and Shouci Lu, Acidized sodium silicate an effective modifier in fluorite flotation Minerals Engineering Volume 5, Issues 3-5 , March-May, Pages 435-444 (1992)

7. Aydın I., Aydın F., Ziyadanoğulları R., Enrichment of U, Mo, V, Ni and Ti from asphaltite ash, Journal of Minerals \& Materials Characterization \& Engineering, Vol. 4, No.1, pp 1-10 (2005) 
8. Ziyadanoğulları R., Aydın F., A New Application For Flotation Of Oxidized Copper Ore, Journal of Minerals \& Materials Characterization \& Engineering, Vol. 4, No. 2, pp 67-73 (2005)

9. Akgün A., Recovery of precious elements from fluorite ore, October, pHD Thesis, Dicle University, Diyarbakir-TURKEY (2002).

10. Vogel A.I., A text book of quantitative inorganic analysis, 3 rd Edn (EIBS Longman, London), 356 (1971). 